\title{
Megalitismo en Évora: musealizar e interpretar
}

Manuel Calado, Dpto. de História, Universidade de Lisboa. Leonor Rocha, Dpto. de História, Universidade de Évora

Traducción: Isabel Moreno Figueiredo

\section{$\cos x$

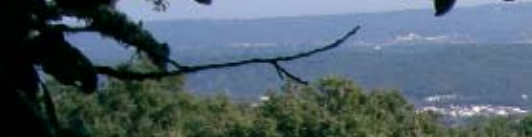

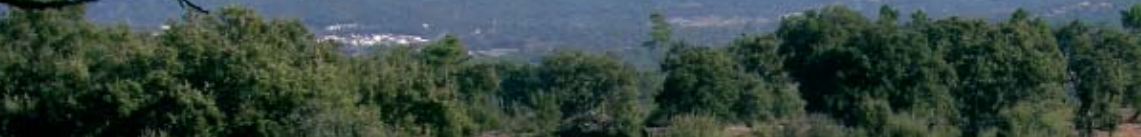

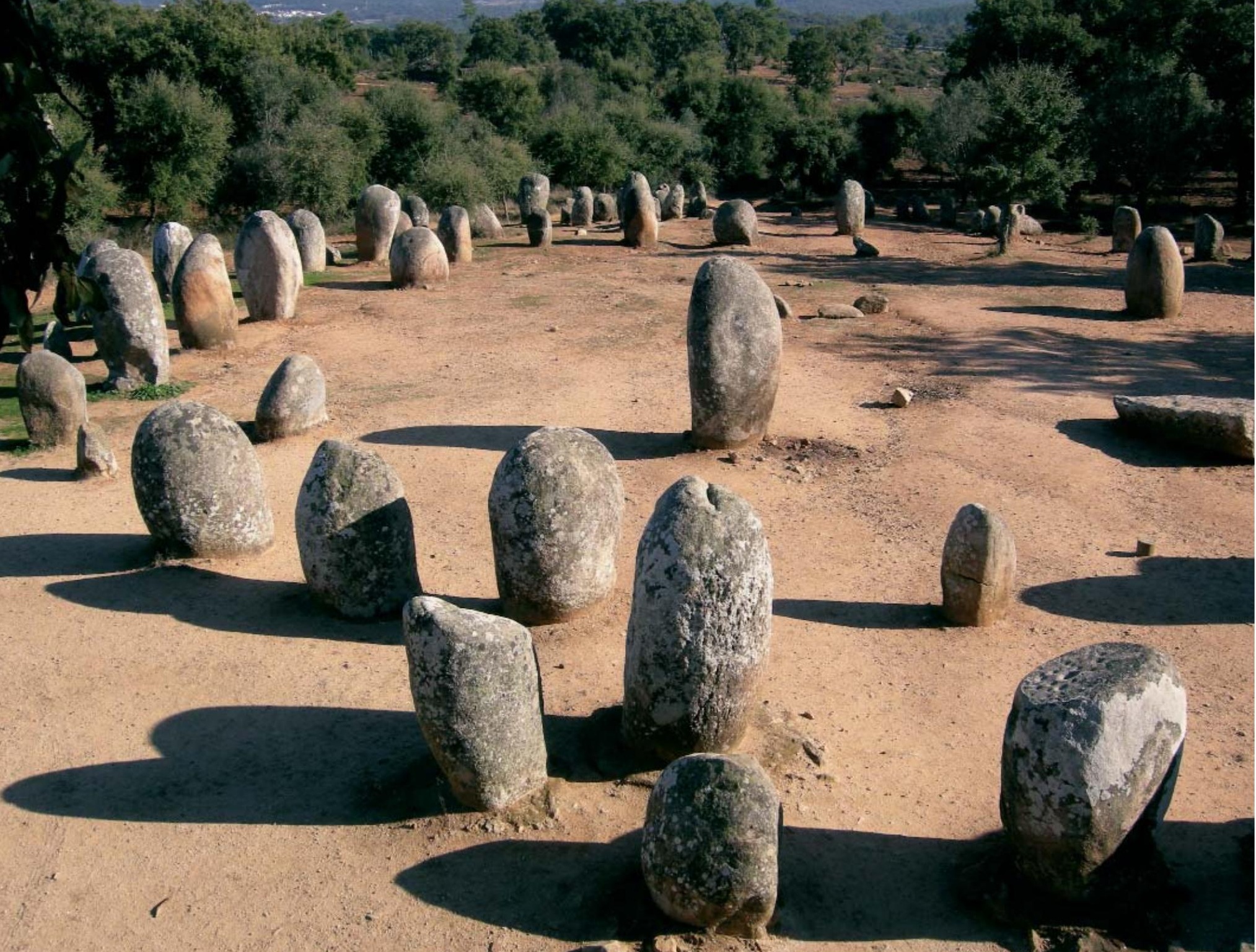




\section{PUNTO DE PARTIDA: IDENTIFICACIÓN Y ESTUDIO}

Los dólmenes eborenses comenzaron desde muy pronto a ser conocidos por los estudiosos, de hecho, algunos fueron incluidos inmediatamente en las primeras recopilaciones efectuadas en Portugal, todavía en la primera mitad del siglo XVIII. La lista de esos monumentos fue significativamente ampliada en la segunda mitad del siglo XIX, y sistematizada, a mediados del siglo XX, gracias al esfuerzo desarrollado por la pareja Georg y Vera Leisner.

En realidad esa labor ha continuado, con altibajos, hasta a nuestros días: en una estimación muy subjetiva, podemos considerar que, excluyendo de este cálculo los monumentos completamente aniquilados, se encuentran registrados cerca del 80 \% de los ejemplares existentes. Quedan, sobre todo, por descubrir algunos de los dólmenes más deteriorados y algunos de los monumentos estructuralmente más discretos, ya sean las sepulturas protomegalíticas, las sepulturas de falsa cúpula o los hipogeos. Sin embargo, no deja de ser curioso que el monumento más extraordinario, dentro de esta categoría no haya sido descubierto hasta los años sesenta del siglo XX: se trata del Dolmen Grande del Zambujeiro, el dolmen más alto del mundo, cuyos ortostatos miden cerca de $8 \mathrm{~m}$ de altura.

En cuanto a los menhires que, hoy día, son el principal icono del Alentejo megalítico, tardaron demasiado en ser identificados: sólo con el descubrimiento del recinto de los Almendres, a mediados de los años sesenta, y de los otros que le siguieron, el tema comenzó a cobrar las dimensiones que hoy le reconocemos. Ciertamente, el primer menhir conocido al sur del Tajo, y uno de los primeros publicados en Portugal, fue descubierto por Gabriel Pereira y publicado en 1880. Ese texto, que pasó desapercibido para la investigación arqueológica subsiguiente fue, de hecho, publicado en una revista generalista que, muy probablemente, tuvo más impacto público que las pocas publicaciones especializadas disponibles en la época.
En las dos últimas décadas, más allá de la profundización en el estudio de menhires y dólmenes (concretamente en aspectos esenciales, como son, a todos los efectos, las cronologías), el conocimiento del megalitismo regional fue bastante ampliado en nuevas direcciones. Destaca, en particular, el registro y el estudio de un elevado número de poblados generalmente contemporáneos del fenómeno megalítico, y el descubrimiento de un importante complejo rupestre en el Guadiana, hasta entonces desconocido. Hubo igualmente avances conceptuales, reflejando, de hecho, las nuevas agendas de la investigación sobre el megalitismo europeo, como es el caso de las diferentes formas de reutilización de menhires en dólmenes o, más generalmente, los diversos fenómenos de continuidad y reinterpretación ocurridos en épocas posteriores a la construcción de los monumentos. Ha ganado también alguna notoriedad en los últimos años la temática de las relaciones simbólicas entre megalitos y paisajes y, con resultados muy sugerentes, el estudio de la orientación astronómica de los monumentos.

\section{CONSERVACIÓN Y RESTAURACIÓN}

A pesar de un panorama razonablemente dinámico en el campo de la investigación, no ha habido, hasta hoy, un programa consistente de conservación y restauración de los monumentos megalíticos de Évora, aunque en los últimos años hayan sido implementadas algunas acciones casuísticas. En los dólmenes excavados, de forma general tuvo lugar, en el mejor de los casos, la reposición de los sedimentos exhumados y, en alguna ocasión, la reimplantación de ortostatos en las respectivas posiciones originales.

En el Dolmen Grande del Zambujeiro, cuyo proceso fue, desde el inicio, un tanto azaroso, algunas señales de degradación estructural 

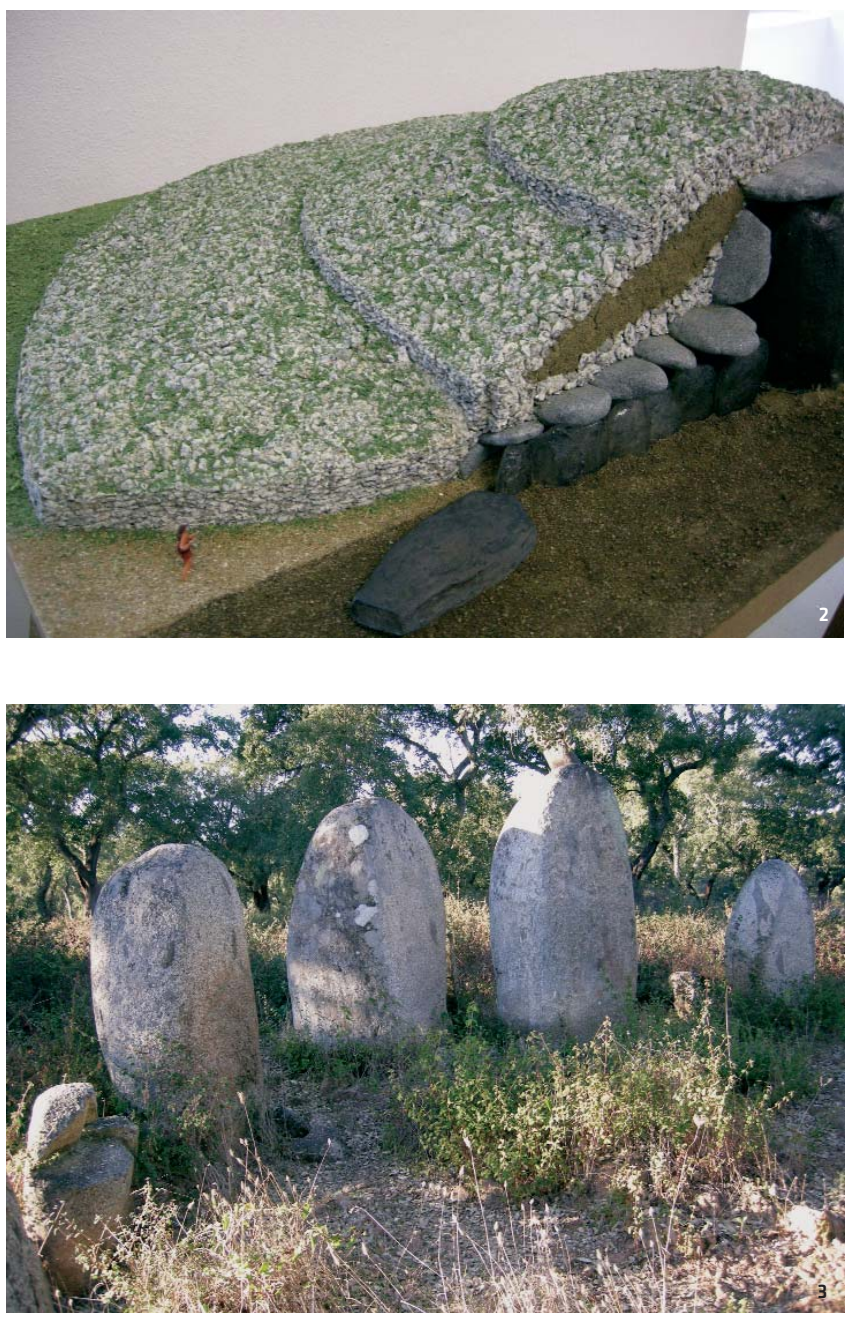

Ilevaron a las autoridades que lo tutelan a instalar, ya en los años ochenta, una cobertura provisional que, desgraciadamente, se mantiene hasta nuestros días. Más recientemente nuevas evidencias de inestabilidad dieron lugar al bloqueo del pasillo con barrotes de madera, impidiendo el acceso a la cámara funeraria. Ciertamente, en la primera intervención de que fue objeto, en la secuencia del descubrimiento, se había efectuado la unión y reimplantación de uno de los ortostatos de la cámara.

En cuanto a los menhires, tampoco ha habido ninguna intervención propiamente dicha en materia de conservación, más allá de la reposición de las tierras excavadas. Sin embargo, al contrario que los dólmenes, estos monumentos han sido objeto de operaciones de restauración relativamente extensas (anastilose), incluyendo la unión de bloques partidos.

\section{MUSEALIZACIÓN, DIVULGACIÓN E INTERPRETACIÓN}

A partir de mediados de los años setenta comenzaron a surgir una serie de itinerarios específicamente dirigidos a la divulgación del megalitismo regional. El primero de estos trabajos, publicado sólo en portugués, en la revista A Cidade Évora, tenía como marco geográfico el distrito de Évora y fue, paralelamente, distribuido bajo la forma de separata. En esta propuesta de Itinerario se incluyeron 35 monumentos: dólmenes, recintos menhires aislados y un afloramiento natural.

Aproximadamente una década después fue publicado un pequeño folleto en portugués, inglés y francés, editado por la Cámara Municipal de Évora, que proponía la visita a un número bastante más reducido de monumentos (diez yacimientos megalíticos más la Gruta del Escoural), manteniendo igualmente una delimitación geográfica a escala de distrito. Ya a finales de esa década, la Cámara Municipal de Évora volvió a editar un nuevo folleto denominado Circuitos Turísticos - Itinerario Megalítico, también trilingüe, que reducía aún más el número de monumentos propuestos y, esta vez, también el territorio comprendido; de hecho, este guión englobaba sólo parte de los municipios de Évora y de Montemoro-Novo y contemplaba únicamente cinco monumentos, uno de los cuales era, una vez más, la Gruta del Escoural. Fue también en esta fase cuando se efectuaron las excavaciones sistemáticas que culminaron con la restauración del recinto megalítico de los Almendres.

La tendencia minimalista se invirtió radicalmente con la publicación, en 1992, de un nuevo Itinerario del Megalitismo de Évora; en este caso, fueron incluidos 25 monumentos, organizados en tres circuitos, centrados en la ciudad de Évora pero abarcando también algunas construcciones del municipio de Montemor-o-Novo, entre ellas la Gruta del Escoural. El proyecto implicó la organización y señalización de los accesos, así como algunos arreglos mínimos, concretamente la limpieza de la vegetación; téngase en cuenta que, a esas alturas, ya se habían introducido importantes mejoras en el acceso al recinto megalítico de los Almendres, que es, aún hoy, el más visitado de todos los monumentos megalíticos alentejanos.

A mediados de los años noventa, la Cámara Municipal de Évora lanzó un nuevo conjunto de guiones, titulados genéricamente Itinerarios Históricos, centrados en el patrimonio de la ciudad de Évora, en diversas épocas, uno de ellos dedicado específicamente al patrimonio megalítico del municipio. En este trabajo se retomó nuevamente una estrategia de reducción de la oferta, intentándose por primera vez un abordaje integrado en el que se incluyeron, dentro del paquete de nueve monumentos a visitar, dos poblados prehistóricos (Alto de S. Bento y Castelo do Giraldo). Paralelamente se colocaron en los dos monumentos más visitados -Zambujeiro y Almendres- paneles con textos explicativos. 
Finalmente, en 2005, fue editado, también por la Cámara Municipal de Évora, un nuevo folleto, en formato de bolsa. En éste se mantuvo y, en cierto modo, se profundizó en el carácter integrado y concentrado del guión anterior. Fueron efectivamente seleccionados sólo cuatro yacimientos/monumentos: el recinto de los Almendres, el menhir del Monte de los Almendres, el Dolmen Grande del Zambujeiro y el poblado del Alto de S. Bento. Con esta propuesta, cuantitativamente muy contenida, esencialmente se pretendía proporcionar el paquete básico para el conocimiento de las diversas modalidades del megalitismo regional: recintos megalíticos, menhires aislados, dólmenes y poblados.

\section{PROYECTOS EN CURSO}

En el municipio de Évora están actualmente en curso dos proyectos de musealización del patrimonio megalítico. A pesar de ser completamente autónomos y protagonizados por instituciones autónomas, los itinerarios propuestos pueden funcionar en muchos aspectos como complementarios: hacen referencia, naturalmente, a monumentos bastante diferentes y, simultáneamente, responden también a conceptos diferentes. El primero, del que es responsable la Cámara Municipal de Évora, está en el orden de las experiencias antes referidas e incluye, como es de esperar, los monumentos más espectaculares que son, de hecho, los más conocidos y demandados por los visitantes. El otro, promovido por la Fundación Eugenio de Almeida, hace referencia a un conjunto muy coherente de sitios, presumiblemente relacionados, localizados en una de las propiedades de la referida Fundación.

\section{Centro de Interpretación del Megalitismo de Évora}

Este proyecto resulta de la colaboración entre la Cámara Municipal de Évora, el Centro de Arqueología de la Facultad de Letras (Uniarq) y la Facultad de Bellas Artes de la Universidad de Lisboa y pretende concretar una estructura interpretativa, de carácter general e integrador, y tiene como objetivo prioritario proporcionar información actualizada, presentada de forma gráficamente atrayente, de los monumentos más simbólicos arriba referidos: el recinto de los Almendres, el menhir del Monte de los Almendres, el Dolmen Grande del Zambujeiro y el poblado del Alto de S. Bento; todos ellos, a excepción de parte del Alto de S. Bento, están localizados en propiedad privada. La elección de este paquete se hizo, como decimos, con base a la monumentalidad/excepcionalidad de los monumentos, pero también, como es natural, a la accesibilidad y, coherentemente, al hecho de ser, ya hoy, por diversas razones, los más visitados.

Ciertamente, la principal (pero no la única) laguna sufrida por los visitantes del Zambujeiro o los Almendres es la falta de información mínimamente
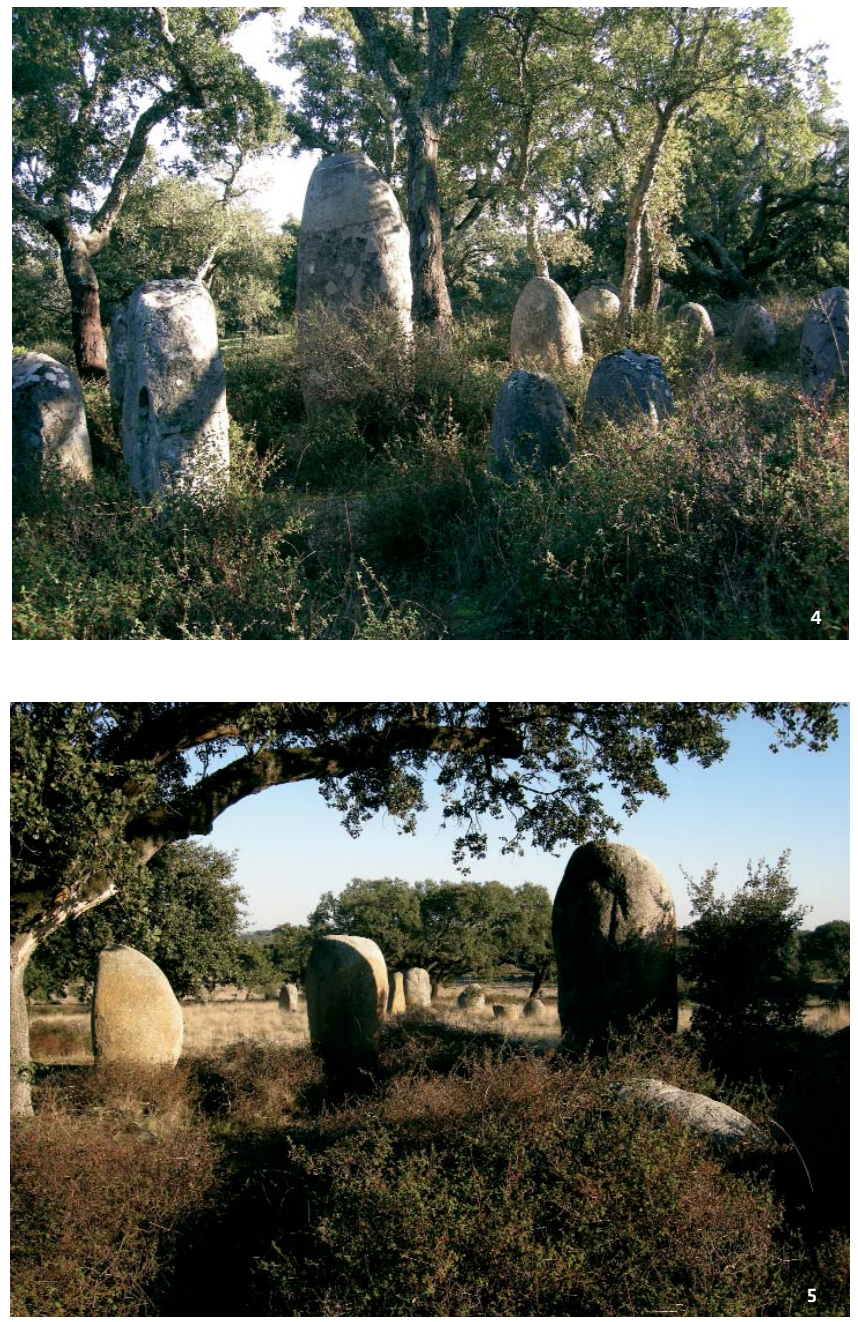

contextualizada sobre el marco, que permita entender unos monumentos fundamentales desde el punto de vista de los especialistas y que, en cualquier caso, no dejan a nadie indiferente. En esta fase la prioridad es, precisamente, instalar en cada uno de los sitios propuestos un panel interpretativo con los datos elementales referentes a cada caso concreto y, a la vez, con información genérica sobre los otros de la misma familia existentes en la región, aunque no estén en condiciones de ser visitados.

Como complemento fundamental se instalará en la ciudad de Évora, en el Convento de los Remedios, un centro interpretativo que pretende constituir un punto de partida, o de llegada, para quien visita los sitios que integran el itinerario. En vez de una estructura informativa sobre cada monumento, se ha optado por un espacio expositivo, que se pretende dinámico, donde el megalitismo regional será contextualizado en dos vertientes principales: por un lado, en la panorámica general del me- 

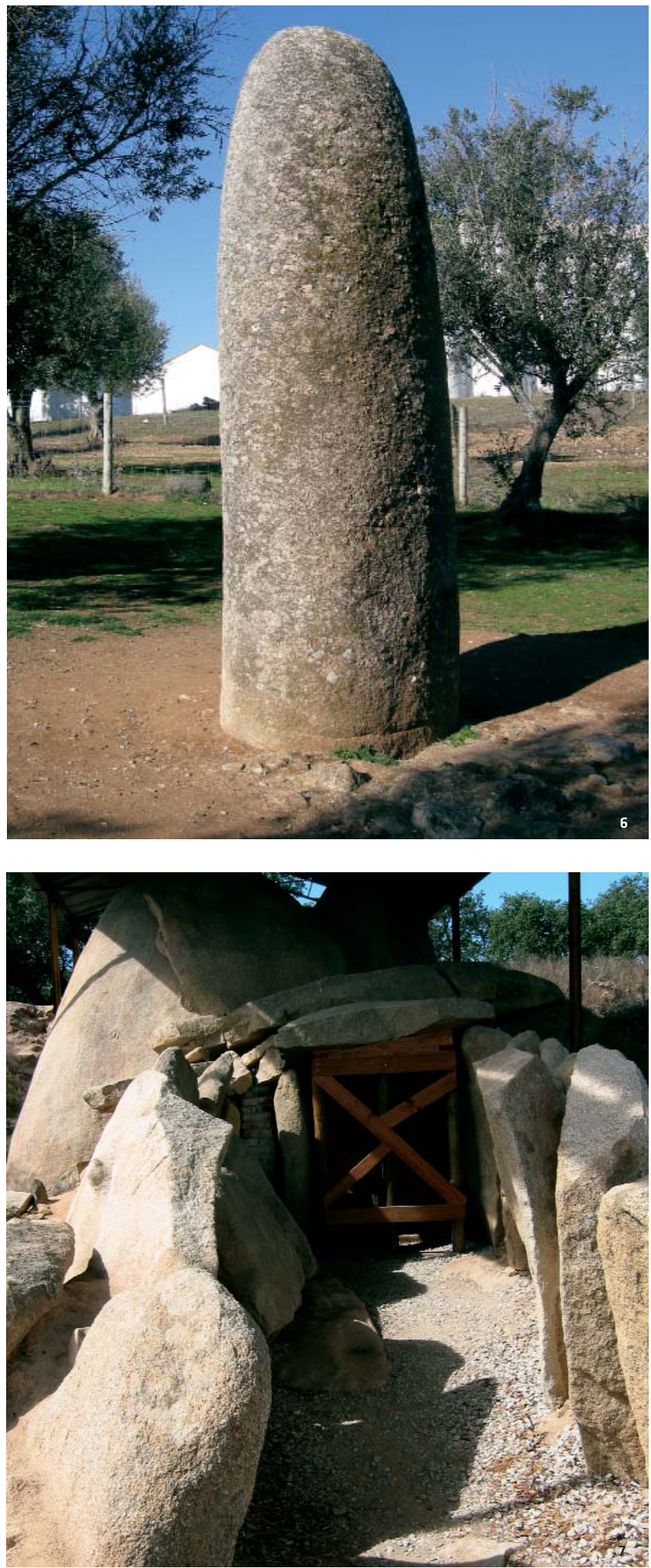

galitismo de la Europa Atlántica, el marco geográfico con que los monumentos alentejanos encuentran más afinidades; y por otro lado, en la época que se produjo en primera instancia: el Neolítico. Este discurso se asienta en la caracterización cultural del Neolítico y, principalmente, de la transición del Mesolítico al Neolítico y de los profundos cambios que ésta implicó.

De principio a fin se pretende profundizar, de una forma simple, accesible y fundamentalmente visual en la cuestión de las orientaciones astronómicas de los monumentos, uno de los aspectos más sugerentes del comportamiento simbólico de esas antiguas sociedades campesinas. Para ello se montará un espectáculo multimedia que funcionará de alguna manera como un planetario, pero centrado exclusivamente en las observaciones de los movimientos de nacimiento del Sol y de la Luna en el horizonte; reproduciendo, por lo tanto, el carácter fenomenológico de la astronomía megalítica.

\section{Conjunto Megalítico de las Murteiras}

Este proyecto presenta características muy particulares. Promovido por una fundación con fines culturales, se encuadra en un programa más amplio de musealización del patrimonio de esta institución, y se centra en un conjunto megalítico articulado con aquel que es, a buen seguro, uno de los paisajes rurales mejor conservados, en el Alentejo central.

Destaca, como elemento principal el poblado neolítico de las Murteiras, donde ha sido recientemente identificada una "cantera" de extracción de bloques megalíticos, en un afloramiento de granito monumental. Más allá de eso, incluye un dolmen y dos sepulturas protomegalíticas, particularmente bien conservadas; en una de las sepulturas ha sido docu-

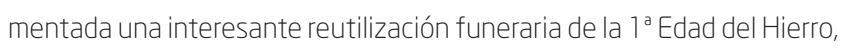
y en el dolmen existen vestigios de reutilización en época romana.

Se trata, objetivamente, de un Itinerario que contextualiza el megalitismo funerario desde una perspectiva cronológica (los antecedentes de los dólmenes y las reutilizaciones tardías) y lo relaciona con un hábitat contemporáneo. Éste se implanta en un paisaje intacto, marcado por afloramientos de granito enormes, con formas redondeadas y caprichosas, estableciendo un puente conceptual entre la elección de los paisajes megalíticos, como lugares de hábitat, y la construcción de monumentos, utilizando elementos de esos mismos paisajes.

\section{BALANCE}

El Alentejo se perfila como una región con fuerte vocación turística, con una cartera de grandes inversiones previstas para los próximos años, que 
promete alterar radicalmente, para bien y para mal, el panorama actual. Los datos apuntan ya el 2007 como un año con fuerte crecimiento turístico. El megalitismo regional es, naturalmente, un recurso de alta calidad en un turismo que se asienta en la valoración de los paisajes y de la cultura. Este escenario implica, como siempre, ventajas y riesgos. La experiencia reciente nos demuestra que la oferta turística de un número demasiado elevado de monumentos es, naturalmente, difícil de gestionar, atendiendo sobre todo al esfuerzo de contención financiera que se vive actualmente: mantener accesos, señalética, limpieza, estructuras informativas; sólo será viable, con dignidad, en un número restringido de sitios. Por otro lado, abrir demasiado la oferta, implica colocar bajo presión a más monumentos.

Una estrategia global, deberá pasar siempre por la inversión pública en los monumentos consensualmente más importantes y por la respectiva disponibilidad de cara a los visitantes, de forma sostenida; paralelamente, se perfila hoy la posibilidad de acciones de puesta en valor de yacimientos y conjuntos megalíticos, de la responsabilidad de entidades privadas, sobre todo en la perspectiva de la respectiva rentabilización turística.

En cualquier caso, muchos centenares de monumentos (son cerca de 900 a escala del distrito) quedarán siempre fuera de acciones concretas de musealización. Éstos están, en su mayoría, disponibles en publicaciones más o menos especializadas o en sitios web, exigiendo del eventual visitante algún esfuerzo de información previa; muchos son susceptibles de "descubrimiento" accidental, siendo visibles desde las carreteras que cruzan la región. Construir itinerarios que propongan "más de lo mismo" no beneficia ni los turistas ni los monumentos.

Abordamos aquí el tema de la puesta en valor del megalitismo sólo desde la óptica del municipio de Évora; en las otras áreas megalíticas alentejanas, sobre todo en Vive Mora o en Reguengos de Monsaraz, existen igualmente monumentos de primer orden, que vienen siendo objeto, los últimos años, de intervenciones puntuales llevadas a cabo por las respectivas cámaras municipales o por la Región de Turismo de Évora, pero en los que la esfera privada comienza a tener un papel cada vez más activo. El paso siguiente, en una perspectiva que se pretende integrada, será la articulación, en términos de promoción turístico-cultural, con las regiones megalíticas adyacentes, en primer lugar, y en escala europea, en segundo. 